\title{
Metodología de Enseñanza en Cursos de Logística para Programas de Administración de Empresas
}

\author{
Jose A. Cano ${ }^{1 *}$ y Carlos J. Ayala ${ }^{2}$
}

(1) Universidad de Medellín, Facultad de Ciencias Económicas y Administrativas, Cra. 87 \# 30-65, Medellín, Colombia. (e-mail: jacano@udem.edu.co)

(2) Fundación Universitaria CEIPA, Escuela de Administración, Calle 77 Sur No. 40 - 165, Medellín,

Colombia. (e-mail: carlos.ayala@ceipa.edu.co)

${ }^{*}$ Autor a quien debe ser dirigida la correspondencia

Recibido Jun. 25, 2018; Aceptado Ago. 30, 2018; Versión final Oct. 2, 2018, Publicado Abr. 2019

\section{Resumen}

Este artículo presenta una metodología de enseñanza aplicando enfoques de aprender-haciendo y servicioaprendizaje basada en la caracterización y mejoramiento de procesos logísticos para la formación logística de administradores de empresas. Esto con el objeto de responder a las necesidades reales de las empresas, y permitiendo la apropiación de conocimientos y competencias brindadas en cursos universitarios de pregrado. Luego de su implementación, se determina a través de encuestas a estudiantes que la metodología propuesta es pertinente y coherente para su uso en el ejercicio profesional. Se observa que la metodología desarrolla competencias para solucionar problemas reales en empresas, e igualmente afianza y pone en práctica los conocimientos aprendidos en gestión logística y otras asignaturas de administración de empresas.

Palabras clave: enseñanza superior; metodología educativa; administración; educación; empresas

\section{Teaching Methodology in Logistics Courses for Business Administration Programs}

\begin{abstract}
This article aims to present a teaching methodology based on learning-doing and service-learning approaches for logistics training in business managers. This with the aim of responding to the real needs of companies and allowing the appropriation of knowledge and skills offered in undergraduate university courses. Through student surveys, it is determined that the proposed methodology is relevant and consistent for use in the professional practice. The methodology develops competencies to solve real problems in companies, and also strengthens and puts into practice the knowledge learned in logistics management and other subjects of business administration.
\end{abstract}

Keywords: higher education; teaching methodology; management; education; enterprises 


\section{INTRODUCCIÓN}

La educación superior enfrenta cada día el reto de formar profesionales con las habilidades y competencias que exige el mercado laboral. El sector de la logística no es ajeno a esto, por lo cual, los cursos de educación en gestión logística deben ajustarse a las necesidades empresariales, aún más si se tiene en cuenta que los empleadores han aumentado su preferencia hacia los profesionales con formación logística (Tong, 2011). Adicional a esto, la gestión de cadena de suministro que incluye la gestión, se ha convertido en una disciplina propia, hasta el punto de liderar los campos en los cuales la administración de empresas se desarrolla, haciendo inevitable que los profesionales en administración reciban educación en gestión logística, y obtengan un rendimiento académico adecuado en su formación universitaria (Tomás-Miquel, Expósito-Langa y Sempere-Castelló, 2014). Para esto, debe entenderse que las cadenas de suministro se han convertido en un elemento clave para incrementar la productividad y competitividad de las compañías, ofreciendo beneficios tanto a las organizaciones, proveedores y clientes (De Camargo y Chiappetta, 2017), debido a que representa el conjunto de actividades funcionales asociadas con el flujo de los productos, servicios, información, finanzas y transformación de bienes desde la etapa de materias primas, hasta llegar al usuario final (Boon-itt, Wong y Wong, 2017). Teniendo en cuenta que la competencia global ha forzado a las empresas a optimizar sus procesos logísticos, esto implica que una adecuada administración de la cadena de suministro debe equilibrar la oferta y demanda de cada uno de sus eslabones a través de los procesos logísticos (Chan, Liu y Szeto, 2017). Por lo tanto, la gestión logística debe minimizar los costos y tiempos relacionados con los movimientos y almacenamientos de productos, desde la adquisición de materiales hasta la entrega de productos terminados a los usuarios finales, cumpliendo a su vez con la promesa de producto, lugar, cantidades, calidad, costo y tiempo pactadas con el cliente (Ou, Arinez, Chang y Xiao, 2017; Cano, Correa-Espinal y Gómez Montoya, 2018).

En este orden de ideas, la gestión logística debe garantizar la supervivencia, crecimiento, productividad y competitividad empresarial a través del buen funcionamiento de las operaciones y procesos de abastecimiento, gestión de proveedores, planificación de la demanda, almacenamiento, gestión de inventarios, manejo de materiales, empaque y embalaje, diseño de redes logísticas, transporte, distribución, cumplimiento de órdenes, gestión de proveedores de servicios logísticos (3PL), servicio al cliente, y flujos inversos para recuperar valor de productos (Banomyong, Veerakachen y Supatn, 2008). En concordancia, las instituciones de educación superior deben desarrollar habilidades relevantes que requieren los administradores de cadenas de suministro para que enfrenten los retos futuros, dentro de las cuales se resaltan las áreas de gestión de almacenes, planificación de la distribución, pronóstico de demanda, habilidades de negociación, coordinación de funciones transversales, conocimiento de problemas y asuntos ambientales en cadenas de suministro, y liderazgo directivo (Rahman y Qing, 2014). Por otra parte, el sector logístico está en constante crecimiento a nivel mundial, hasta el punto de representar uno de los empleos con mayor potencial, especialmente para el personal calificado como técnico logístico y administrador en logística (Erturgut, 2011). Esta situación ha llevado a que la educación logística sea protagonista en los índices de planes logísticos regionales en diferentes países, y a que las empresas soliciten mayores esfuerzos de sus gobiernos para aumentar la educación en logística, hasta el punto de reevaluar la prioridad y presupuesto que se tiene para la educación y entrenamiento en logística, con el fin de mejorar así la operación logística en ámbitos empresariales y humanitarios (Bölsche, Klumpp y Abidi, 2013).

En este sentido, las universidades son el escenario ideal para el desarrollo de diversos enfoques en logística y educación en administración (Canen y Canen, 2001), y están llamada a preservar la identidad profesional de la logística dentro y fuera de las escuelas de administración, evitando que la educación logística sea diluida dentro de otras asignaturas universitarias y disciplinas profesionales que no tienen una verdadera orientación hacia la gestión de cadena de suministro (Zinn y Goldsby, 2014). Por lo tanto, los administradores de empresas están llamados a liderar los procesos logísticos, debido a que las competencias en gestión son esenciales para que los administradores logísticos sean exitosos, y esto genera el reto de redefinir los estándares de las competencias en logística, incluyendo enfoques multidisciplinarios y de sostenibilidad, que integren capacidades técnicas, económicas, administrativas y gerenciales (Marinov y Fraszczyk, 2014). La educación superior está cambiando para que los estudiantes sean quienes participen activamente en el proceso de formación de competencias, y el profesor asuma un rol de acompañamiento y mediador (Marinov y Fraszczyk, 2014). Esto lleva a que el enfoque de la educación en gestión logística y de cadena de suministro se centre en al aprendizaje activo, donde se combinan elementos de simulación y práctica que ayudan involucrar de mejor forma a los estudiantes al proceso de aprendizaje. Igualmente, se destaca que los estudiantes deben asumir el reto de incorporar los conocimientos y casos de estudio vistos en clase en un ambiente de aprendizaje virtual (modelación y simulación), soportado por tecnologías de información y comunicación (Marín, Inciarte, Hernández, y Pitre, 2017; Rodriguez, Ramirez, y Basile, 2017), y en un ambiente real (acercamiento a empresas), donde se conduzca a la experiencia de aprender haciendo (Rodriguez, Ramirez y Fernández, 2017), y sea posible realizar una evaluación del aprendizaje específico y de las soluciones que generan los estudiantes. Adicional a esto, las actividades de formación y enseñanza en 
logística basadas en equipos de trabajo permiten a los estudiantes poner en práctica sus aprendizajes dentro de ambientes profesionales, enriqueciendo su formación a través de la retroalimentación y aval de sus compañeros en la toma de decisiones (Hummel, Hyra, Ranz y Schuhmacher, 2015).

Para el logro de una educación logística de calidad, los profesores deben contar con mecanismos de aprendizaje y metodologías de enseñanza adecuados que permitan mejorar la adquisición y retención de conocimiento (Lu, Goh y De Souza, 2013), y que a su vez garanticen que la formación del recurso humano en logística esté compuesta por experiencia empresarial, habilidades sociales, de toma de decisiones, de solución de problemas, de gestión de tiempo y prioridades, y de conocimientos generales, tal como lo demanda el sector industrial y empresarial (Lutz y Birou, 2013). En consecuencia, los profesores en gestión logística deben adoptar varias metodologías de aprendizaje activo, entre los cuales se pueden mencionar proyectos en empresas y proyectos de investigación, que permiten la acumulación de experiencia, articulación y codificación de, conocimiento, fortalecen la construcción de un concepto sólido de gestión logística a través de la visualización de cómo funciona la logística en una realidad empresarial, y ayudan a generar ventaja competitiva sustentable en empresas logísticas. Todo esto invita a que las empresas en las cuales se enfoquen los trabajos de los estudiantes en gestión logística se conviertan en fábricas de aprendizaje, en donde se realiza una transferencia de las mejores prácticas académicas y prácticas, favoreciendo las relaciones universidad-empresa (Hummel et al., 2015; Chan-Pavon, Mena-Romero, Escalante-Euán, y Rodríguez-Martín, 2018).

De esta forma, la educación en gestión logística esta llamada al diseño de cursos y métodos que hagan posible desarrollar conocimientos y habilidades en los estudiantes, con lo cual, se beneficien también facultades, comunidades e instituciones a través de la aplicación de enfoques de aprender-haciendo o enfoques de servicio-aprendizaje, donde a medida que desarrollan soluciones logísticas por parte de los estudiantes, estos brindan un servicio a las empresas en estudio, ofreciendo posibles soluciones y herramientas para mejorar sus procesos (Lu et al., 2013). Este acercamiento de los estudiantes a las empresas disminuye la brecha que existe entre las empresas y las universidades, debido a que los empresarios y educadores suelen tener enfoques diferentes sobre las habilidades y temáticas críticas que deben priorizarse en un currículo en logística para un administrador logísticos (Wu, Huang, Goh, y Hsieh, 2013). Con base en lo expuesto anteriormente, este artículo tiene como objetivo desarrollar una metodología de enseñanza basada en la caracterización mejoramiento de procesos logísticos para la formación logística en administradores de empresas, con el fin de guiar a los educadores para la implementación de mecanismos que permitan que los estudiantes den respuestas a las necesidades reales de las empresas, y que permitan la apropiación de conocimientos y competencias brindadas en cursos universitarios de pregrado.

\section{PROPUESTA METODOLÓGICA PARA LA FORMACIÓN EN GESTIÓN LOGÍSTICA}

Con base en los aportes identificados en la literatura, se presenta en este apartado una propuesta metodológica para la enseñanza en gestión logística, que permite que los estudiantes apliquen los conocimientos y aprendizajes en ambientes reales, proponiendo una solución técnica y económicamente viable en empresas. Esta metodología busca que los equipos de trabajo de estudiantes de administración de empresas puedan caracterizar los procesos logísticos de empresas de manufactura o prestación de servicios, y tengan la competencia de identificar las principales oportunidades de mejora, para proponer de esta forma proyectos que permitan mejorar la productividad y competitividad empresarial a través de procesos logísticos.

El enfoque metodológico propuesto pretende que los estudiantes sean los protagonistas del proceso de aprendizaje al aplicar sus conocimientos adquiridos en logística en escenarios reales. De esta forma, los estudiantes tienen contacto directo con las empresas, y reciben orientación del profesor en cada etapa de la metodología, asegurando que el proceso de aprendizaje a través de la práctica sea efectivo. En adición, la propuesta metodológica se sustenta en herramientas basadas en MSExcel, y herramientas informáticas de propósito general que son aplicados fácilmente en ambientes de formación de gestión logística (Jiang, 2010).

\section{Selección de la empresa de estudio}

La metodología de aprendizaje en logística comienza con la selección de una empresa, la cual puede ser de cualquier tamaño (pequeña, mediana, grande) y puede dedicarse a la fabricación de productos o prestación de servicios. Lo más importante en el proceso de selección de empresa, es que la empresa pueda brindar toda la información posible respecto a los procesos logísticos que lleva a cabo. Luego, se realiza una descripción básica de la empresa, donde se evidencia la misión, visión, valores, clientes, portafolio de productos y servicios, entendiendo así el contexto corporativo de la empresa. 


\section{Caracterización de procesos logísticos}

La caracterización de los procesos logísticos de la empresa se orienta con la guía que se presenta en la Tabla 1, donde se establecen los principales procesos logísticos a analizar, y las operaciones dentro de dichos procesos. Para cada operación logística se sugieren algunas temáticas principales para facilitar a los estudiantes la obtención de la información logística necesaria. Se resalta que en cada proceso logístico abordado en la caracterización de la Tabla 1, se analizan las tecnologías de información y comunicación (TIC) que soportan dichos procesos, ya que estas tecnologías representan recursos necesarios para obtener beneficios de la integración de procesos logísticos dentro y entre los eslabones de una cadena de suministro.

Tabla 1: Guía para realizar la caracterización de procesos logísticos

\begin{tabular}{|c|c|c|}
\hline Proceso & Operaciones & Temáticas principales \\
\hline \multirow[t]{5}{*}{$\begin{array}{l}\text { Planificación } \\
\text { Logística }\end{array}$} & $\begin{array}{l}\text { Pronósticos de } \\
\text { demanda }\end{array}$ & $\begin{array}{l}\text { Métodos de pronósticos de demanda. Confiabilidad de los pronósticos de } \\
\text { demanda. }\end{array}$ \\
\hline & $\begin{array}{l}\text { Capacidad de los } \\
\text { procesos }\end{array}$ & Cálculo de la capacidad de los sistemas logísticos. Planes de contingencia. \\
\hline & Operadores logísticos & Procesos logísticos a tercerizar, selección y seguimiento de proveedores 3PL. \\
\hline & $\begin{array}{l}\text { Capacitación de } \\
\text { personal }\end{array}$ & $\begin{array}{l}\text { Capacitaciones en logística. Frecuencia y proveedores de capacitaciones en } \\
\text { logística. }\end{array}$ \\
\hline & Tecnologías (TIC) & Tecnologías y software, y su pertinencia para la planificación logística. \\
\hline \multirow[t]{4}{*}{ Compras } & $\begin{array}{l}\text { Gestión de materias } \\
\text { primas }\end{array}$ & Clasificación y control de calidad de suministros. Centralización de compras. \\
\hline & $\begin{array}{l}\text { Gestión de } \\
\text { proveedores }\end{array}$ & Caracterización, clasificación, selección y evaluación de proveedores. \\
\hline & Políticas de compra & Políticas de compra. Cantidades y frecuencia de compras. \\
\hline & Tecnologías (TIC) & Tecnologías y software, y su pertinencia para el proceso de compras. \\
\hline \multirow{4}{*}{$\begin{array}{l}\text { Gestión de } \\
\text { Inventarios }\end{array}$} & Conteo de inventarios & Frecuencia de conteos de inventarios. Confiabilidad de inventarios \\
\hline & Niveles de inventarios & Cobertura y rotación de inventarios. Averías y obsoletos de inventarios. \\
\hline & $\begin{array}{l}\text { Clasificación de } \\
\text { inventarios }\end{array}$ & Clasificación ABC de inventarios, y políticas a cada clase de inventario. \\
\hline & Tecnologías (TIC) & Tecnologías y software, y su pertinencia para gestión de inventarios. \\
\hline \multirow[t]{3}{*}{$\begin{array}{l}\text { Gestión de } \\
\text { almacenes }\end{array}$} & Sistemas del almacén & $\begin{array}{l}\text { Infraestructura y zonas de almacenamiento, eficiencia en operaciones y } \\
\text { espacios. }\end{array}$ \\
\hline & Manejo de materiales & Equipos de manejo de materiales dentro del almacén. \\
\hline & Tecnologías (TIC) & Tecnologías y software, y su pertinencia para el proceso de almacenamiento. \\
\hline \multirow[t]{4}{*}{$\begin{array}{l}\text { Distribución } \\
\text { y Transporte }\end{array}$} & \begin{tabular}{|l|} 
Sistemas de \\
transporte
\end{tabular} & Medios y modos de transporte, cubicaje, seguimiento y trazabilidad de carga. \\
\hline & $\begin{array}{l}\text { Distribución de } \\
\text { productos }\end{array}$ & $\begin{array}{l}\text { Programación y ruteo para la distribución de productos. Indicadores de servicio } \\
\text { al cliente. }\end{array}$ \\
\hline & $\begin{array}{l}\text { Canales de } \\
\text { distribución }\end{array}$ & $\begin{array}{l}\text { Redes y canales de distribución. Eficiencia y eficacia de los canales de } \\
\text { distribución. }\end{array}$ \\
\hline & Tecnologías (TIC) & Tecnologías y software, y su pertinencia para el proceso de distribución y transporte. \\
\hline \multirow[t]{4}{*}{$\begin{array}{l}\text { Logística } \\
\text { Inversa }\end{array}$} & $\begin{array}{l}\text { Devoluciones y } \\
\text { recambios }\end{array}$ & Atención de devoluciones, rechazos y reposiciones a los clientes. \\
\hline & Sostenibilidad & Recuperación y reciclaje de materiales. Disminución de impacto ambiental. \\
\hline & Servicio posventa & Servicio posventa y de garantías de productos para los clientes. \\
\hline & Tecnologías (TIC) & Tecnologías y software, y su pertinencia para el proceso de logística inversa. \\
\hline
\end{tabular}

Una vez se obtiene la información de la descripción cualitativa de las operaciones logísticas, esta se ingresa en una plantilla de MSExcel, y se complementa con el ingreso de sugerencias y oportunidades de mejora para cada operación analizada. De esta forma los estudiantes en una etapa inicial obtienen un contexto global de los procesos logísticos de la empresa, y las posibles soluciones o proyectos de mejora que podrían aplicarse. Con la descripción cualitativa de las operaciones logísticas de la empresa, se procede a asignar una valoración cuantitativa a cada una de estas. Dicha valoración varía entre 0 y 5 , y los valores asignados dependen de cómo la empresa en estudio lleva a cabo sus procesos empresariales, y qué tan acordes son con el tamaño de la empresa, volumen de operaciones, nivel de ventas de la empresa. En la Tabla 2 se muestra los rangos de valoración cuantitativa para asignar a las operaciones logísticas y la equivalencia o significado de estos valores. Se aclara que es posible que dentro de algunas empresas no se apliquen algunas logísticas a causa de la naturaleza o razón de ser de la empresa. En estos casos no se asigna una calificación numérica a la operación y se asigna una valoración con el término N/A (No aplica), con el fin de no afectar la calificación de los procesos logísticos y de la gestión logística como tal. 
Tabla 2: Valoración cuantitativa para las operaciones logísticas

\begin{tabular}{|c|l|}
\hline $\begin{array}{c}\text { Escala de } \\
\text { calificación }\end{array}$ & Equivalencia \\
\hline 0 a 1 & Deficiente aplicación en la empresa de estudio \\
\hline 1 a 2 & Regular aplicación en la empresa de estudio \\
\hline 2 a 3 & Buena aplicación en la empresa de estudio \\
\hline 3 a 4 & Muy buena aplicación en la empresa de estudio \\
\hline 4 a 5 & Excelente aplicación en la empresa de estudio \\
\hline N/A & No aplica para la empresa de estudio \\
\hline
\end{tabular}

\section{Selección del proceso y operación logística a mejorar}

Teniendo en cuenta la valoración numérica asignada a cada operación logística, se facilita la selección del proceso y operación logística a mejorar. La calificación obtenida para cada proceso logístico se calcula como un promedio aritmético de calificaciones las operaciones logísticas que pertenecen a dicho proceso. Luego, con base en la calificación obtenida para cada proceso logístico, se calcula el desempeño global de la gestión logística como el promedio de la calificación de los seis procesos logísticos tenidos en cuenta. De esta forma, se tiene un criterio claro y coherente para seleccionar y priorizar las operaciones logísticas a intervenir y mejorar, debido a que el proceso logístico con menor calificación será el proceso a intervenir, especialmente en la operación que presentó menor puntaje. Esto significa que se mejorará la operación que más impacto genera en el desempeño de todo el sistema logístico de la empresa.

\section{Descripción de la operación logística seleccionada}

Para describir la operación logística a mejorar, se sugiere el uso de una matriz SIPOC (Supplier, Input, Processes, Output and Customer), que permite describir el objetivo, alcance, proveedores, entradas, actividades, salidas y proveedores de una determinada operación (Salazar, Gomez, y Cano, 2017). La descripción de la operación logística también se complementa con la descripción de indicadores, sistemas de información, recursos, y con el uso de diagramas de flujo, imágenes, diseños, videos y demás documentación de soporte que describa en detalle cómo se lleva a cabo la operación logística seleccionada en la empresa de estudio.

\section{Propuesta de mejora logística}

La propuesta de mejora a la operación logística seleccionada, debe ajustarse a las falencias identificadas previamente en la descripción de la operación. Estas propuestas de mejora pueden surgir de la aplicación de conocimientos, técnicas, modelos y metodologías aprendidas durante las sesiones de clase del curso de gestión logística, así como también pueden obtenerse de diversas fuentes bibliográficas donde se presenten casos de estudio o soluciones pertinentes al problema abordado en la operación logística. Desde el punto de vista técnico, la propuesta de mejora debe detallarse a profundidad, para comprender su implementación e impactos esperados. Con el fin de obtener el plazo en el cual se ejecutará e implementará la propuesta, se debe desarrollar un cronograma, detallando en un diagrama de Gantt cada actividad a desarrollar, los responsables de las actividades y los entregables o productos que genera cada actividad.

Una vez se establece el tiempo de ejecución e implementación del proyecto de mejora, se procede a realizar la sustentación económica de la propuesta, soportada por una hoja de cálculo en MSExcel. Esta sustentación económica se requiere para demostrar los beneficios de la propuesta a los miembros de la cadena de suministros, y para estimar los ingresos potenciales obtenidos con la mejora de métodos y habilidades de los administradores logísticos (Ballou, 2007). De esta forma puede realizarse un pronóstico de los posibles retornos que puede traer la implementación de proyectos de mejora, que pueden incluir la capacitación de empleados, el cambio de procesos, mejoramiento de infraestructura, implementación de tecnologías, entre otras. De forma similar, se calculan los costos de inversión periódica, gastos fijos y variables que exige la propuesta de mejora; con lo cual se puede calcular el beneficio neto que ofrece la propuesta de mejora en cada periodo. Por último, para determinar la viabilidad económica del proyecto de mejora, se calculan indicadores tales como la tasa de oportunidad del inversionista (TIO), valor presente neto (VNA), tasa interna de retorno (TIR), y periodo de retorno de la inversión (PRI), que sirven como apoyo para determinar si la propuesta de mejora genera los impactos esperados por la empresa de estudio.

\section{Informe final}

Los grupos de trabajo de estudiantes deben presentar un informe gerencial escrito, donde se evidencie cada una de las etapas de la metodología, los principales hallazgos y conclusiones de la propuesta de mejora 
empresarial. Este informe se envía por parte de los estudiantes a la empresa que les facilitó la información para realizar el estudio. Igualmente, se socializan los trabajos de cada equipo de estudiantes en una sesión de clase, a través de presentaciones ejecutivas que deben tener una duración entre 15 a 25 minutos, con el fin de recopilar las percepciones de los estudiantes respecto al trabajo realizado, y establecer la cercanía que se tuvo con la empresa analizada durante la elaboración del trabajo aplicado (Canen y Canen, 2001). Luego de la socialización, los estudiantes presentan conclusiones sobre cuáles son los problemas logísticos más frecuentes en las empresas y cómo estos deben solucionarse.

\section{RESULTADOS}

Para validar la efectividad y satisfacción de la metodología propuesta, se aplicó una encuesta a un total de 448 estudiantes que realizaron el curso de gestión logística dirigido por 2 docentes en un programa de administración de empresas en Colombia, y cursan el último año de estudios. Del total de la población, 247 estudiantes respondieron la encuesta, con lo cual se obtiene un tamaño de muestra válido debido a que al aplicar un muestreo aleatorio simple (MAS) que considera un nivel de confianza del $95 \%$ y un $5 \%$ de error se genera un tamaño de muestra de 207 estudiantes. En la Tabla 3, se resume la encuesta aplicada a los estudiantes para conocer su satisfacción con la metodología de aprendizaje, el fortalecimiento de competencias en logística y oportunidades de mejora de la metodología. La encuesta se realizó de forma virtual a través de Google Forms, omitiendo la identificación del estudiante para asegurar transparencia y sinceridad en las respuestas obtenidas. Adicionalmente, la encuesta fue validada por 4 docentes expertos en logística pertenecientes a la Red de Profesionales en Logística, Operaciones y Negocios (REDPLOYN), que opera en la ciudad de Medellín, Colombia.

Tabla 3: Encuesta sobre la metodología de aprendizaje

\begin{tabular}{|l|l|l|}
\hline \multicolumn{1}{|c|}{ Aspectos } & Preguntas & Tipo de pregunta \\
\hline $\begin{array}{l}\text { Pertinencia y } \\
\text { satisfacción } \\
\text { con la } \\
\text { metodología }\end{array}$ & $\begin{array}{l}\text { ¿Considera pertinente y coherente el uso de la metodología de caracterización } \\
\text { logística y mejoramiento de procesos logísticos para el aprendizaje, desarrollo } \\
\text { y fortalecimiento de competencias en gestión logística? }\end{array}$ & Única respuesta \\
\cline { 2 - 3 } & $\begin{array}{l}\text { ¿En su ejercicio profesional, volvería a emplear o recomendaría el uso de esta } \\
\text { metodología para caracterizar y mejorar procesos logísticos? }\end{array}$ & Única respuesta \\
\cline { 2 - 4 } & $\begin{array}{l}\text { ¿Qué tan satisfecho se encuentra al utilizar la metodología de caracterización } \\
\text { logística y mejoramiento de procesos logísticos? }\end{array}$ & Única respuesta \\
\hline $\begin{array}{l}\text { Desarrollo de } \\
\text { competencias } \\
\text { en logística }\end{array}$ & $\begin{array}{l}\text { ¿Cuáles competencias considera que desarrolla o fortalece la metodología de } \\
\text { caracterización y mejoramiento de procesos logísticos? }\end{array}$ & $\begin{array}{l}\text { Múltiple } \\
\text { respuesta }\end{array}$ \\
\cline { 2 - 4 } & $\begin{array}{l}\text { ¿Cuáles ventajas considera que ofrece la metodología de caracterización y y } \\
\text { mejoramiento de procesos logísticos según su criterio? }\end{array}$ & $\begin{array}{l}\text { Múltiple } \\
\text { respuesta }\end{array}$ \\
\hline $\begin{array}{l}\text { Oportunidades } \\
\text { de mejora }\end{array}$ & $\begin{array}{l}\text { ¿Cuáles oportunidades de mejora considera que deben acogerse para una } \\
\text { mayor eficiencia y eficacia de la metodología? }\end{array}$ & $\begin{array}{l}\text { Múltiple } \\
\text { respuesta }\end{array}$ \\
\hline
\end{tabular}

En la Figura 1 se observa que la mayoría de estudiantes expresan que la metodología es pertinente y coherente, y que están dispuestos a volver a utilizarla en su ejercicio profesional como administradores de empresas. Respecto a la satisfacción con el uso de la metodología para la enseñanza en logística, se identifica en la Figura 2 que el 96,4\% de los estudiantes expresan un alto grado de satisfacción con la metodología, lo cual indica que los estudiantes perciben que la metodología propuesta aporta significativamente a su proceso de formación profesional en gestión logística.

\begin{tabular}{|c|c|c|c|c|c|}
\hline \multicolumn{2}{|c|}{$\begin{array}{l}\text { Pertinencia de la } \\
\text { metodología }\end{array}$} & \multicolumn{2}{|c|}{$\begin{array}{l}\text { Coherencia de la } \\
\text { metodología }\end{array}$} & \multicolumn{2}{|c|}{$\begin{array}{l}\text { Uso de la metodología en su } \\
\text { ejercicio profesional }\end{array}$} \\
\hline \multirow[t]{2}{*}{$99,2 \%$} & & $99,2 \%$ & & $99,2 \%$ & \\
\hline & $0,8 \%$ & & $0,8 \%$ & & $0,8 \%$ \\
\hline $\mathrm{Si}$ & No & $\mathrm{Si}$ & No & $\mathrm{Si}$ & No \\
\hline
\end{tabular}

Fig. 1: Pertinencia y coherencia de la metodología de aprendizaje. 


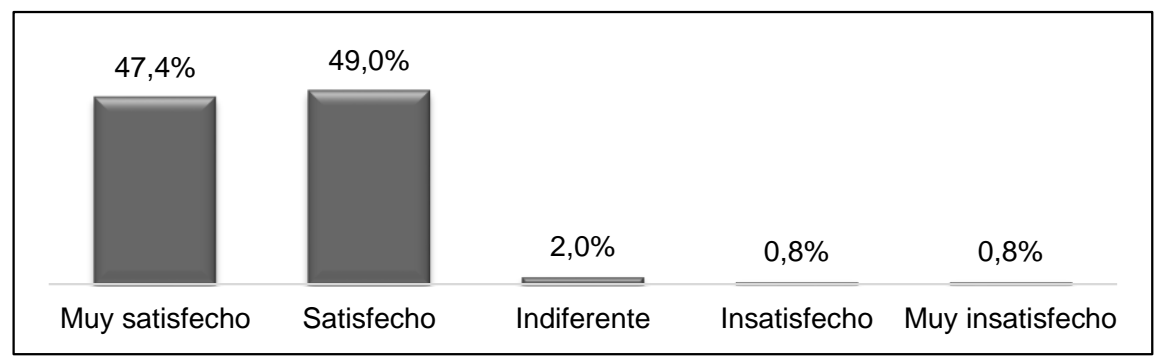

Fig. 2: Nivel de satisfacción con el uso de la metodología.

En cuanto al desarrollo de competencias en gestión logística, la Figura 3 sugiere que la metodología propuesta permite principalmente identificar oportunidades de mejora de procesos logísticos, aplicar los conocimientos vistos en los diferentes módulos de la asignatura a través de un caso real, y proponer mejoras a los procesos logísticos con base en información cualitativa y cuantitativa. Estas competencias en particular presentaron porcentajes iguales o superiores al $40 \%$.

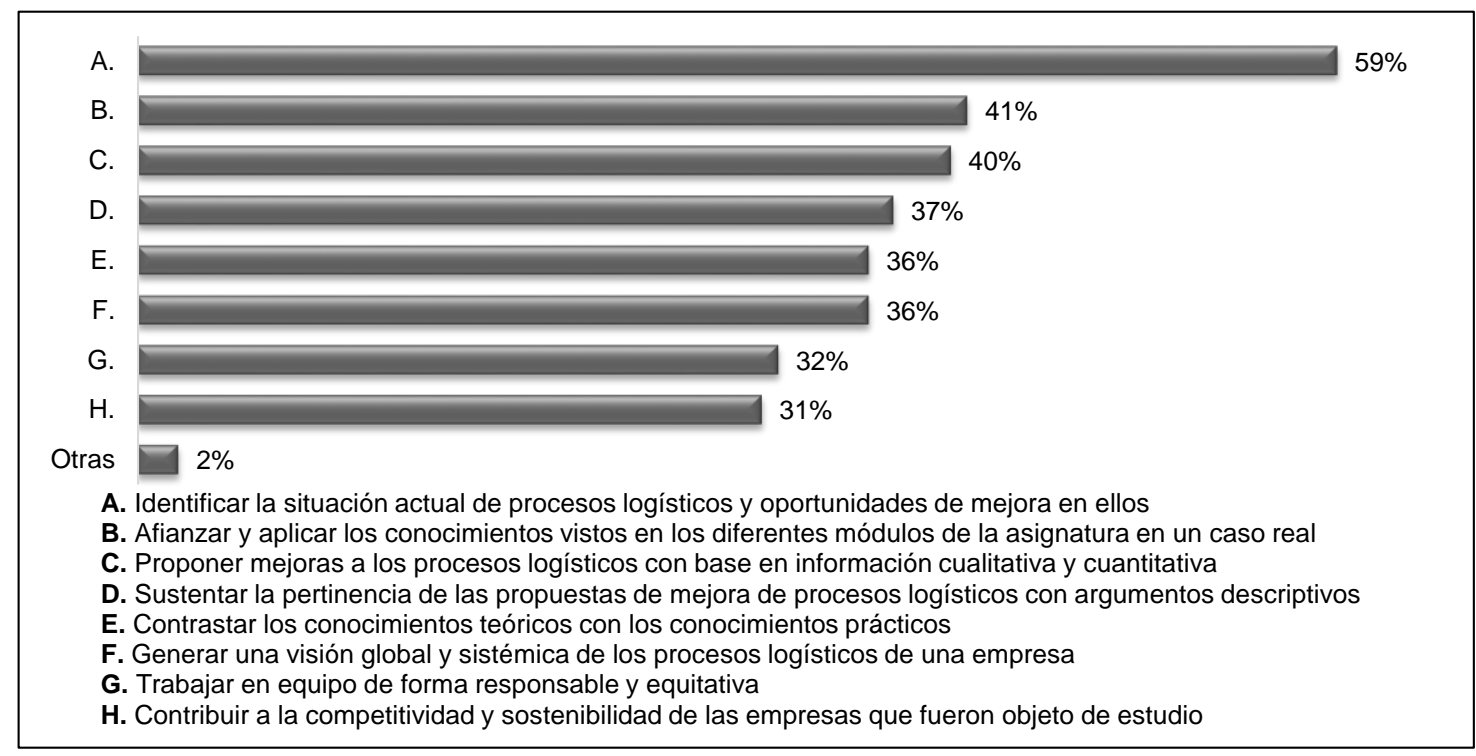

Fig. 3: Competencias profesionales desarrolladas con la metodología.

Adicional a esto, la Figura 4 muestra que los estudiantes de administración de empresas consideran que la metodología genera soluciones a problemas reales con argumentos técnicos y económicos, contacta a los estudiantes con una empresa real y sus directivas, y pone en práctica un conjunto de conocimientos y competencias adquiridas en diferentes asignaturas, incluyendo el curso de gestión logística.

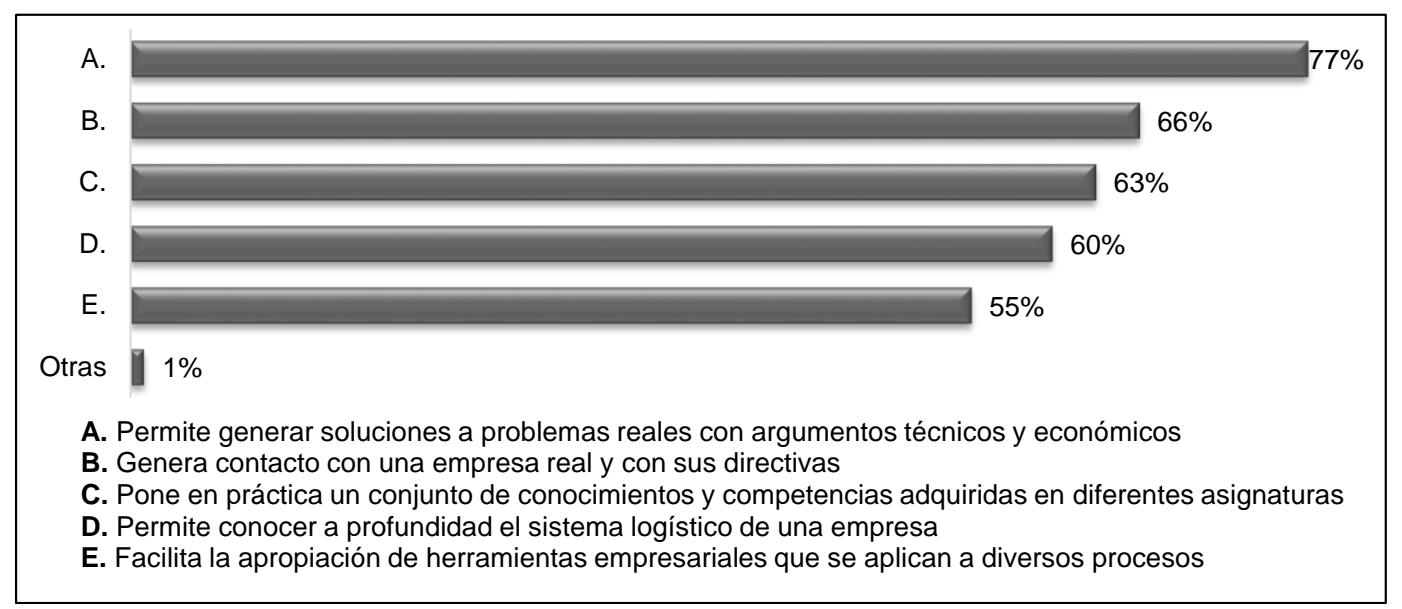

Fig. 4: Ventajas ofrecidas por la metodología.

Por otro lado, la Figura 5 sugiere que en la implementación de la metodología se debe facilitar la búsqueda de empresas, enfatizar en la explicación del uso de las herramientas de valoración económica, y orientar a los estudiantes para coordinar los equipos de trabajo. 


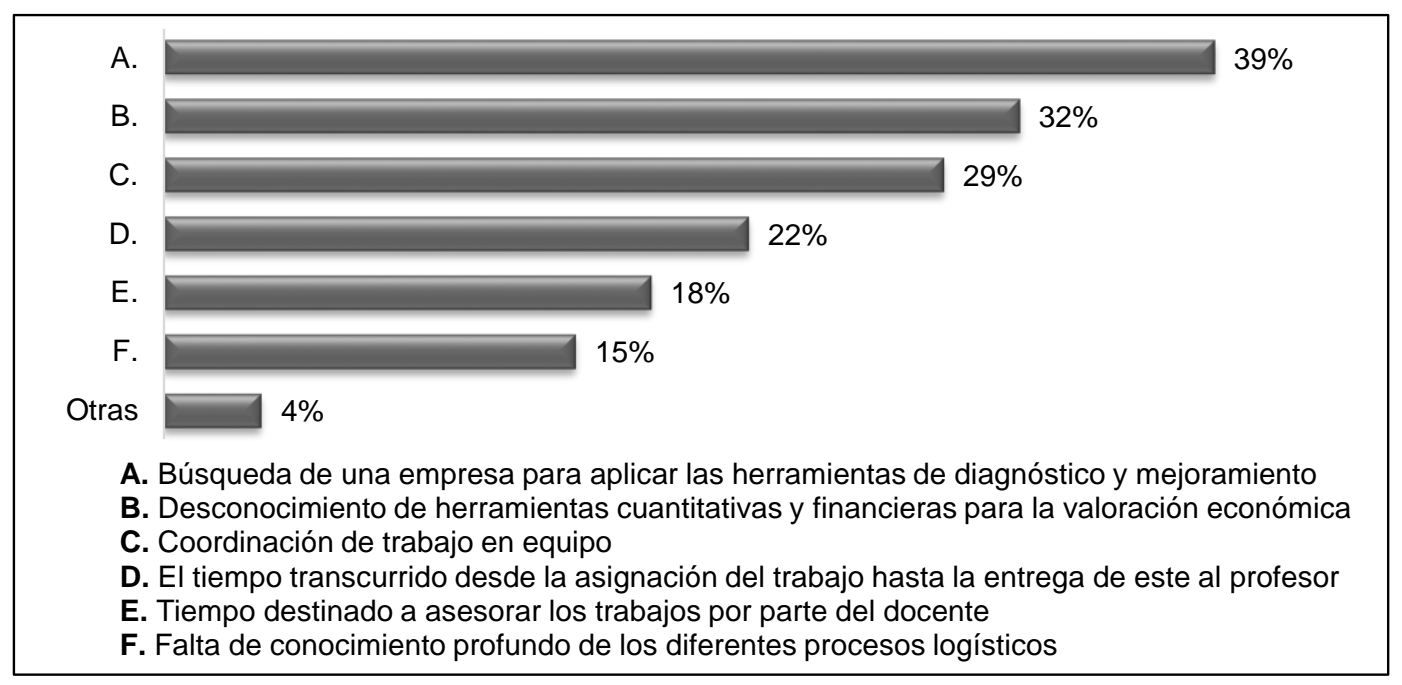

Fig. 5: Oportunidades de mejora de la metodología.

\section{DISCUSIÓN FINAL}

El desarrollo de metodologías de aprendizaje para la gestión logística debe ofrecer un ambiente de aprendizaje cercano a la realidad, basado en conceptos didácticos y metodologías relevantes de formación (Hummel et al., 2015). En concordancia a este planteamiento, en este artículo se presentó una metodología con la cual los estudiantes de administración de empresas identifican sistemáticamente oportunidades de mejora en procesos y operaciones empresariales, con el fin de proponer soluciones y mejoras que sean viables técnica y económicamente. A través de un trabajo colaborativo entre estudiantes se garantiza con la propuesta metodológica que los estudiantes tengan contacto directo con el mundo empresarial, y retroalimenten a las empresas con proyectos de mejora. De esta forma se aplica una metodología basada en el enfoque de aprender-haciendo, donde a través de una experiencia empresarial los estudiantes desarrollan herramientas de diagnóstico logístico y de gestión de proyectos, y aplican un enfoque de servicio-aprendizaje, con el cual se brinda un servicio de caracterización logística a las empresas analizadas, así como también una formulación de proyectos de mejora, validados económicamente, para su posterior implementación. Igualmente, con la metodología propuesta, se incentiva el uso de herramientas informáticas de amplio uso empresarial como el MSExcel, así como la integración de conocimientos de diferentes asignaturas que permiten a los estudiantes universitarios en administración de empresas la construcción de una propuesta de mejoramiento integral.

La metodología propuesta demuestra ser pertinente y coherente para su uso en el ejercicio profesional de los administradores de empresa. Además de esto, ofrece un alto grado de satisfacción en el proceso de formación profesional, y permite a los estudiantes identificar y solucionar problemas reales de procesos logísticos en empresas con argumentos técnicos y económicos. Por lo tanto, la metodología permite que se afiance la relación universidad-empresa, a través de un intercambio de conocimientos que le permite a los estudiantes aprender y apropiarse de herramientas empresariales a través de una práctica real de los conocimientos vistos en clases, y le permite a las empresas recibir una estructuración de sus procesos logísticos y una formulación de propuestas para el mejoramiento continuo de las operaciones empresariales. Finalmente, se recomienda complementar la metodología propuesta con iniciativas por parte de los departamentos de extensión de las universidades, que permitan ejecutar por parte de las universidades los proyectos de mejora identificados en las empresas, dando un avance mayor para materializar las oportunidades detectadas por los estudiantes. De esta forma, se ofrecerá a las empresas un servicio completo de soluciones empresariales desde las universidades, y los estudiantes fortalecerán sus competencias y experiencia en la formulación de proyectos de mejora en logística y cadena de suministro.

\section{CONCLUSIONES}

De acuerdo al trabajo presentado y a los resultados obtenidos, se pueden plantear las siguientes conclusiones principales:

1.- La metodología propuesta para la enseñanza en cursos de gestión logística es pertinente y coherente, genera un alto grado de satisfacción e incentiva a su uso en el ejercicio profesional de administradores de empresas, todo ello gracias a los enfoques de servicio-aprendizaje y aprender-haciendo.

2.- La metodología propuesta identifica oportunidades de mejora de procesos logísticos, aplica los 
conocimientos vistos en el curso de gestión logística, y propone mejoras a los procesos logísticos con base en información cualitativa y cuantitativa. Esto hace se trate de una metodología valiosa y comprehensiva que permite la evaluación de procesos y formulación de acciones de mejoramiento, con lo cual se propicia la construcción de conocimiento.

3.- De esta forma, las propuestas realizadas por los estudiantes permiten solucionar problemas en empresa reales con argumentos técnicos y económicos, afianzando de esta forma el enlace entre universidad y empresas, y brindando soluciones a los requerimientos laborales y profesionales que presentan las empresas productivas.

\section{REFERENCIAS}

Ballou, R.H., The evolution and future of logistics and supply chain management, doi:10.1108/09555340710760152, European Business Review, 19(4), 332-348 (2007)

Banomyong, R., V. Veerakachen y N. Supatn, Implementing leagility in reverse logistics channels, doi:10.1080/13675560701403651, International Journal of Logistics Research and Applications, 11(1), 31-47 (2008)

Bölsche, D., M. Klumpp y H. Abidi, Specific competencies in humanitarian logistics education, doi:10.1108/JHLSCM-082012-0019, Journal of Humanitarian Logistics and Supply Chain Management, 3(2), 99-128 (2013)

Boon-itt, S., C.Y. Wong y C.W.Y. Wong, Service supply chain management process capabilities: Measurement development, doi:10.1016/j.jpe.2017.06.024, International Journal of Production Economics, 193(1), 1-11 (2017)

Canen, A.G. y A. Canen, Looking at multiculturalism in international logistics: an experiment in a higher education institution, doi:10.1108/09513540110384493, International Journal of Educational Management, 15(3), 145-152 (2001)

Canen, A.G., Looking at multiculturalism in international logistics: an experiment in a higher education institution, doi:10.1108/09513540110384493, International Journal of Educational Management, 15(3), 145-152 (2001)

Cano, J.A., A.A. Correa-Espinal y R.A. Gómez-Montoya, Solución del problema de conformación de lotes en almacenes utilizando algoritmos genéticos, http://dx.doi.org/10.4067/S0718-07642018000600235, Información Tecnológica, 29(6), 235-244 (2018)

Chan, C.O., O. Liu y R. Szeto, Developing information sharing model using cloud computing and smart devices for SMEs supply chain: A case in fashion retail, doi:10.4018/IJISSCM.2017070103, International Journal of Information Systems and Supply Chain Management, 10(3), 44-64 (2017)

Chan-Pavon, M.V., D.A. Mena-Romero, J.F. Escalante-Euán y M.D. Rodríguez-Martín, Contribución de las Prácticas Profesionales en la formación de los Estudiantes de la Facultad de Ingeniería Química de la Universidad Autónoma de Yucatán (México), doi:10.4067/S0718-50062018000100053, Formación Universitaria, 11(1), 53-62 (2018)

De Camargo, P. y C.J. Chiappetta, Information systems and sustainable supply chain management towards a more sustainable society: Where we are and where we are going, doi:10.1016/j.ijnfomgt.2016.12.004, International Journal of Information Management, 37(4), 241-249 (2017)

Erturgut, R., Increasing demand for logistics technician in business world and rising trend of logistics programs in higher vocational schools: Turkey case, doi:10.1016/j.sbspro.2011.04.187, Procedia - Social and Behavioral Sciences, 15, 2776 $2780(2011)$

Hummel, V., K. Hyra, F. Ranz y J. Schuhmacher, Competence Development for the Holistic Design of Collaborative Work Systems in the Logistics Learning Factory, doi:10.1016/j.procir.2015.02.111, Procedia CIRP 32(1), 76-81 (2015)

Jiang, C., A reliable solver of euclidean traveling salesman problems with Microsoft Excel add-in tools for small-size systems, Journal of Software, 5(7), 761-768 (2010)

Lu, Q., M. Goh y R. De Souza, Learning mechanisms for humanitarian logistics, doi:10.1108/JHLSCM-10-2012-0031, Journal of Humanitarian Logistics and Supply Chain Management, 3(2), 149-160 (2013)

Lutz, H. y L. Birou, Logistics education: a look at the current state of the art and science, doi:10.1108/SCM-08-2012-0269, Supply Chain Management: An International Journal, 18(4), 455-467 (2013)

Marín, F.V., A.J. Inciarte, H.G. Hernández y R.C. Pitre, Estrategias de las Instituciones de Educación Superior para la Integración de las Tecnología de la Información y la Comunicación y de la Innovación en los Procesos de Enseñanza. Un Estudio en el Distrito de Barranquilla, Colombia, doi:10.4067/S0718-50062017000600004, Formación Universitaria, 10(6), 29-38 (2017)

Marinov, M. y A. Fraszczyk, Curriculum Development and Design for University Programmes in Rail Freight and Logistics, doi:10.1016/j.sbspro.2014.05.198, Procedia - Social and Behavioral Sciences, 141(1), 1166-1170 (2014)

Ou, X., J. Arinez, Q. Chang y G. Xiao, Cost analysis and fuzzy control for collapsible container usage based on closedloop supply chain model, doi:10.1115/1.4036522, Journal of Manufacturing Science and Engineering, Transactions of the ASME, 139(8), 1-11 (2017)

Rahman, S. y N. Qing, Graduate students' perceptions of supply chain skills for supply chain managers, doi:10.1108/BIJ01-2012-0002, Benchmarking, 21(2), 276-299 (2014) 
Rodriguez, A.B., L.J. Ramirez y F.R.M. Basile, Tecnologías y Educación: Su Percepción Social En Santiago de Chile, doi:10.4067/S0718-50062017000600008, Formación Universitaria, 10(6), 67-76 (2017)

Rodriguez, A.B., L.J. Ramirez y W. Fernández, Metodologías Activas para Alcanzar el Comprender, doi:10.4067/S071850062017000100009, Formación Universitaria, 10(1), 79-88 (2017)

Salazar, F., R.A. Gomez y J.A. Cano, El problema de carga de pallets en centros de distribución utilizando diseño de mezclas, Espacios, 38(2), 2 (2017)

Tomás-Miquel, J.-V., M. Expósito-Langa y S. Sempere-Castelló, Determinantes del rendimiento académico en los estudiantes de grado, Un estudio en administración y dirección de empresas, doi:10.6018/rie.32.2.177581, Revista de Investigación Educativa, 32(2), 379-392 (2014)

Tong, J., Managing Logistics Higher Education Using Logical Framework Analysis, International Journal of Innovation, Management and Technology, 2(4), 309-314 (2011)

Wu, Y.C.J., S.K. Huang, M. Goh y Y.J. Hsieh, Global logistics management curriculum: Perspective from practitioners in Taiwan, doi:10.1108/SCM-04-2012-0145, Supply Chain Management, 18(4), 376-388 (2013)

Zinn, W. y T.J. Goldsby, Logistics professional identity: Strengthening the discipline as galaxies collide, doi:10.1111/jbl.12038, Journal of Business Logistics, 35(1), 23-28 (2014) 